\title{
Normalization Techniques for Translating Cultural - Specific Expressions
}

\author{
Nuchanad Imjidee* \& Soh Bee Kwee \\ Faculty of Languages and Linguistics \\ University of Malaya, Malaysia \\ Submitted: 14/06/2020. Revised edition: 09/08/2020. Accepted: 15/11/2020. Published online: 30/11/2020
}

\begin{abstract}
For readability of audience in target culture (TC), cultural-specific expressions (CSEs) which have been embedded with specific characteristics, need specific techniques to transfer them into target language (TL). This study aims to identify normalization techniques (NTs) from domestication strategies to show that they are particularly necessary for CSE translation. Based on the previous studies of different scholars, the overlap between domestication and normalization is clarified, following by the clarification of the relation between normalization and the use of translator's subjectivity, as well as the distinction between CSEs and universals for simple explanation on what normalization and CSE are. Last but not least, the overlapping NTs, classified from domestication strategies will be unified. Finally, illustration of normalization of CSEs, selected from Thai target text (TT) and its English source text (ST), The Da Vinci Code (DVC), a novel by Dan Brown, will give an overt explanation of how each NT is used to deal with CSEs in order to show relation between characteristics of CSEs and each NT. This will answer why NTs are necessary.
\end{abstract}

Keywords: Normalization technique, domestication strategy, cultural-specific expression, the Da Vinci Code, normalized CSEs

\subsection{INTRODUCTION}

In 2010, Erika Mussche and Klaas Willems studied the transfer of cultural-specific items (CSIs) in Harry Potter from English into Arabic. What they focused were the relation between procedures and cultural properties in the translation process and effect of the applied procedures on the TT, and at the end they found that normalization plays an important role on the translation of CSIs. How is normalization important in culture-specific translation? "[...], normalization has featured among socalled universals since the beginning, so to speak, i.e., since Baker $(1993,1996)$ drew her list of likely candidates to that consideration. However, it had already been identified as a trait of translated text not universally but in specific environments [...]" (Marco, J., 2009: 845). This clearly asserts that NT is particularly used for transferring CSE. CSE is a term, phrase, or sentence with sense,

\footnotetext{
*Correspondence to: Nuchanad Imjidee (email: neverdown2525@gmail.com)
} 
connotation, or even history that is perceived among usersof source language (SL) in source culture (SC). Each NT itself has specific function to solve the difficulties, caused by the gathered properties of each CSE. This study will show that each NT correlates with CSE as a solution for a problem.

\subsection{LITERATURE REVIEW AND DISCUSSION}

\section{Difference between Domestication and Normalization}

In 2012 Azizollah Dabaghi and Mohammad Bagheri attempted to explore which solution(s) was used in rendering CSE in a literary text (Blind Owl) from Persian into English and found that the translator domesticated the ST's CSEs by using cultural equivalence and paraphrasing (using generic word and descriptive phrase). Also, it was found that when it was necessary to look over meaning and equivalence, the substitution of SL's expressions with TL's which served the same function was inevitable to be used, 'functional equivalence' could preserve the same impression with ST in TT, and paraphrasing was used to convey the intended meaning sometimes when SL's figurative expressions cannot be rendered into TL.

The study on the process of translating cultural elements (CEs) in Oliver Twist by Charles Dickens from English into Arabic, Tarek Atallah Saleh Al-Idhesat (2016: 3-4) aimed to reveal (1) CEs used in the Arabic translation (2) the translation procedures employed to preserve the qualities and features of the CEs in the TT and (3) whether the CEs have been domesticated or foreignized, based on the use of the type of translation procedure. Based on Vinay and Darbelnet's seven translation procedures (1995) which were categorized into two types: (1) direct translation (borrowing, calque and literal translation) and (2) oblique translation (equivalence, transposition, adaptation and modulation), the study discovered that the most majority CEs are rendered by oblique translation, but some by direct. Finally, it was found that most of the CEs were domesticated into TT.

Back in 2012, Rowena Coles analysed English-Italian translation of Children's novel, Once by Morris Gleitzman. She defined 'domestication' as 'a way to bring a text through translation closer to culture of target readers and defined 'normalization' as a use of unmarked language in a specific context to tend towards textual conventionality, whether in syntactical or lexical level. From the study, she concluded that in the translation of this novel, in general, domestication strategy had been used, through normalization process, to adapt the original text in order to make it more acceptable to the TL readers. Under domestication strategy being employed, extracted examples from the translation text revealed that there were three phenomena taking place in the normalization process including (1) change in syntax (2) normalization of lexical creativity (3) change in register. And each phenomenon revealed as follows:

1. Change in syntax:

- modality in ST had not been transmitted. Mood, marked form and complexity in ST disappeared and TT had weaker impact than ST consequently. 
- Simple sentences had become compound sentences in target version leading to a loss of pause of the original.

- Marked elliptic forms of the original sentences had been reintroduced in TT.

- Upgraded structure of ST was reinstated by omission of subjects and verbs, resulting in disappearance of its stylistic expressivity in target version.

- The repetition and the abrupt sentences in the original were not used in TT, but were articulated into a more complex syntactical form. As a result, TT had lost the tone of the original.

2. Normalization of lexical creativity:

- Tone in the original, being awarded with a notable degree of lexical creativity and easily distinguishable idiolect was toned down, as well as striking originality and strong impact.

- The expression of striking lexical creativity, providing linguistic entertainment, in SL, was paraphrased and explicated in two coordinated clauses, but its impact was lessened in TT.

- Normal TL terms were used for marked SL terms which caused a decrease of stylistic expressivity of the original.

3. Change in register: this phenomenon was probably the one that leaded to the most significant changes in the translation. Referring to Michael Halliday, register is described as "variety according to use" (1985: 43), for example, vocabulary in the original sentence was transformed from typical language with humorous touch to genteelism to make the scene more genteel in TT, but the humorous touch had gone.

With reference to the text itself, firstly, it showed that marked sentence structures had often undergone a normalization process, and that unusual lexical choices had been replaced by more usual unmarked ones. Secondly, a greater use of connectives and more explicitness in the translation had been found which made the text more accessible to the TL readers; however, it led to the removal of challenges offered by the author to the SL readers and the change of atmosphere and creative style of the original. In conclusion, the strategies in the translation were adopted to adapt the text, presumably to make it compatible with the norms governing the TL's literary system and to accommodate it to particular values of the TL's culture.

From all analyses, it has been found that the purpose of domesticating is "bringing the text, through translation, closer to the culture of the implied reader" (Coles, R., 2012: 45). It is the type of translation which is designated by Lawrence Venuti (1995) for minimizing the foreignness of ST for TL readers (e.g. Shuttleworth, M., \& Cowie, M., 1997; Yang, W., 2010; Wang, F., 2014). Regarding to techniques used in domestication, for example, it was found in Azizollah Dabaghi and Mohammad Bagheri's study (2012) that CSEs in a Persian literary text, Blind Owl, were domesticated into English through paraphrasing and substitution with cultural equivalence and functional equivalence, whereas in Al-Idhesat, T. A. S.'s study (2016), most of cultural elements from an English novel, Oliver Twist, were found that had been domesticated into Arabic through oblique translation or the use of equivalence, transposition, adaptation and modulation techniques. 
Meanwhile, normalization, proposed by Mona Baker (1996) as the way of translation conforming to the practices of TL, was elucidated by many scholars such as Kateř ina Olexová (2009), Yun Xia (2014) and Mohamed M. Mostafa (2015) that the main aim of normalization in translation is to orient ST towards patterns, norms or textual conventionality of TL, by minimizing manifestation of SL expressions in ST, for target readers' readability and familiarity. From the above paragraphs, it can be seen that normalization and domestication share the same purpose and the same way and techniques of translation. This conforms to Rowena Coles' statement (2012: 45) that domestication strategy had been used, through normalization process, and Kateř ina Olexová's (2009: 22) that normalization is a technique of domestication.

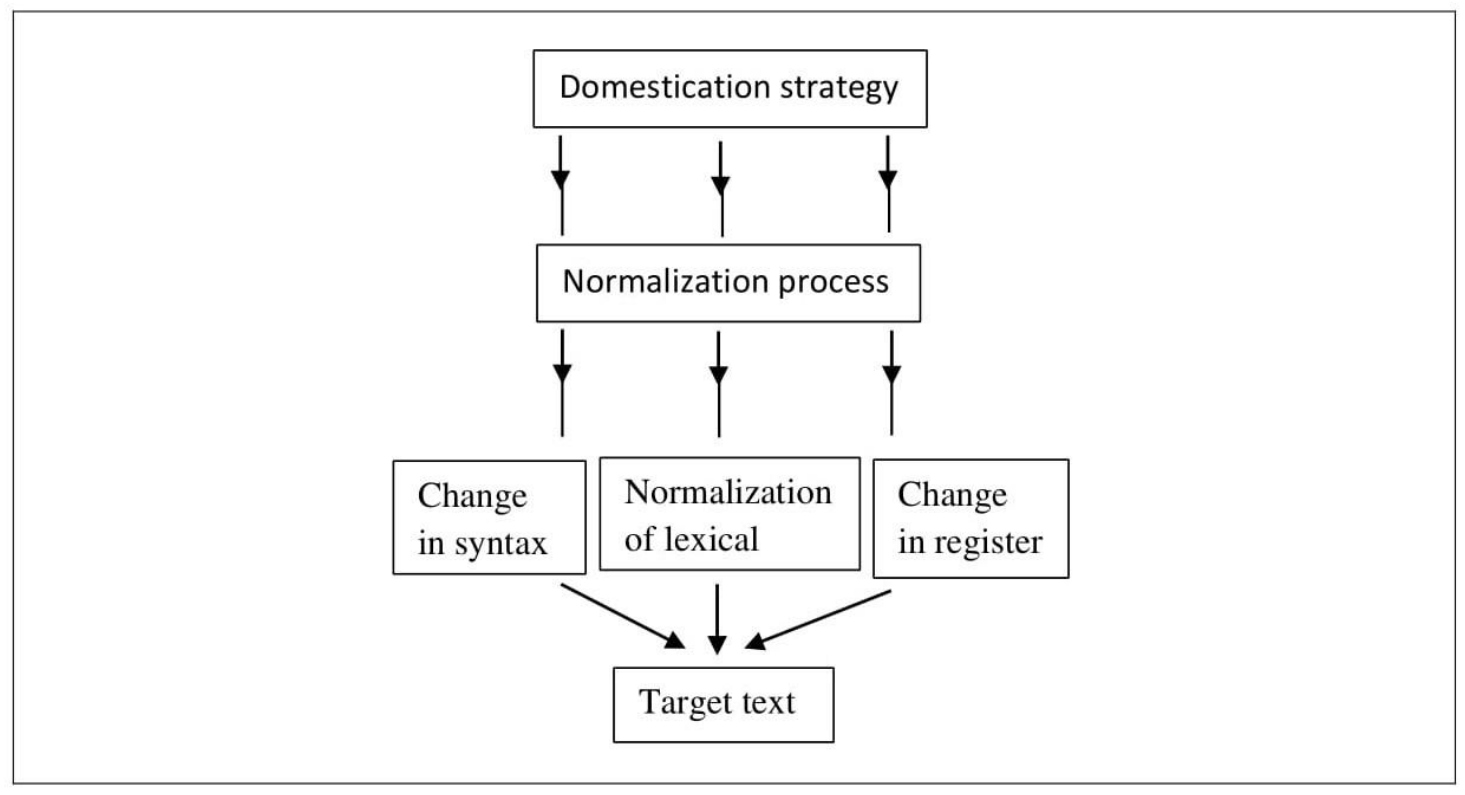

Figure 1 The illustration of Rowena Coles's proposal of a process of normalization

\section{Normalization and Translator's Subjectivity}

As mentioned in the previous study, Implications of Lexical Choices in Translating Novels as Literary Texts, by Jamal Mohamed Giaber (2015: 415-419), the process of literary translation from one language to another, in particular, is govern by linguistic, sociocultural and personal factors. Personal factor relates to translator's subjectivity and idiolect, inherent features in translator as a reader and writer. Subjectivity primarily affects reading and interpreting of translator. As an interlingual and intercultural communicator, automatically, in the process of decoding and encoding, translator uses his/her personal or subjective judgment to re-express the ST ideas in TT. Thus, inevitably, the translator's idiolect is on the surface of translation. This involves lexical, grammatical and stylistic 
choices of the translator, which should reflect the semantic, pragmatic and aesthetic values of the original in TL, however, based on his/her interpretation of SL text. The study had also discussed three notions placing on the implications of translator's word choice: language variation, register and lexical choices, in which register or the use of language according to the situation intimately relates to lexical alternatives.

It appears that translator's word choice, supported by the three notions, reflects in those phenomena in the normalization process, found in the study by Rowena Coles (2012), in particular, the phenomena of change in register. In the way of change in register, a translator chooses words from a variety of language to make a normalization of the translation, by considering task or event that the words are used. That is to say, translator's word choice, based on his/her subjectivity, is a part of normalization process. To recreate an equivalent work of art with the same message, tone and effect of ST in TL is the ultimate goal of the use of normalization process and subjectivity of the translator.

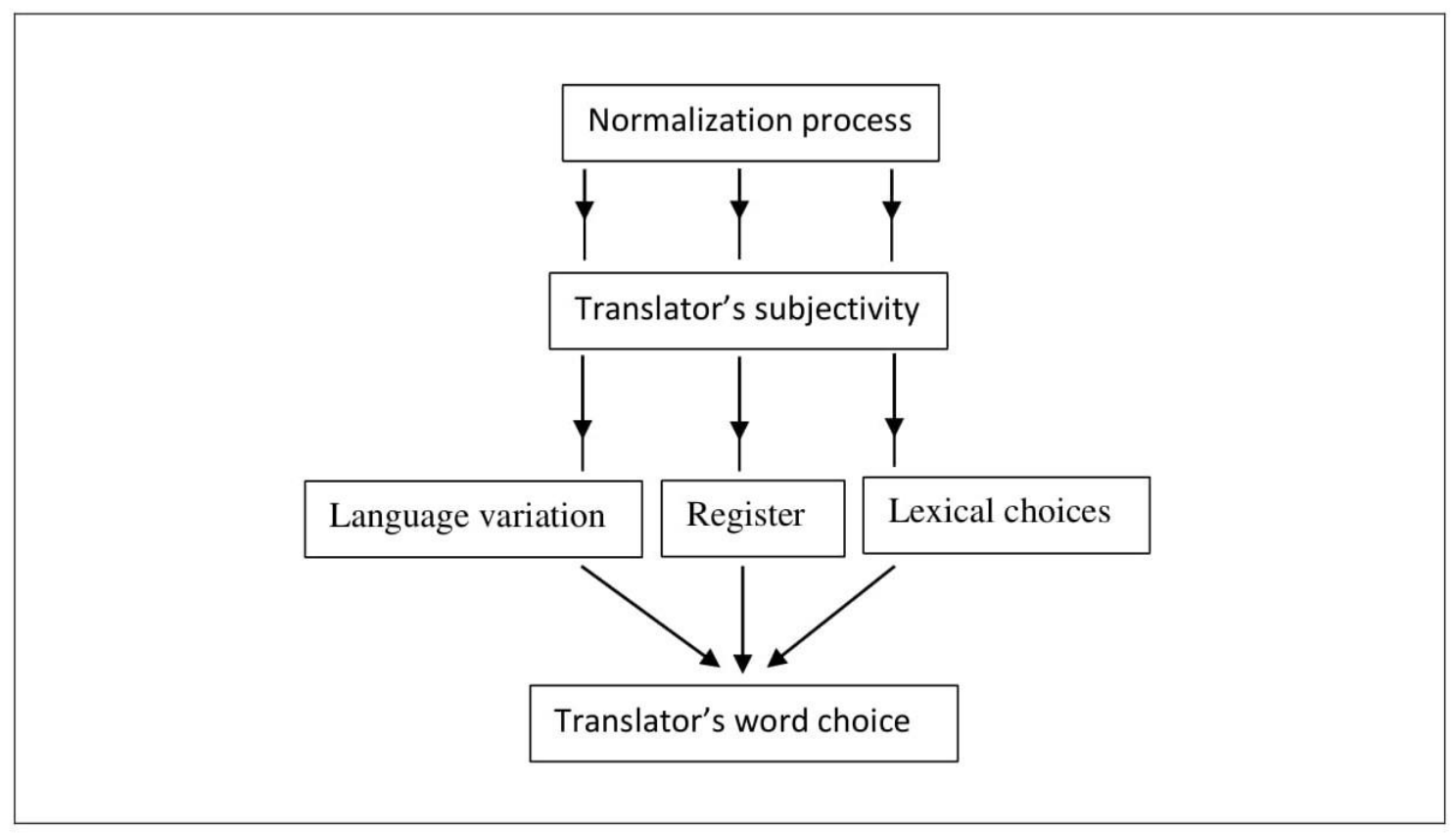

Figure 2 The illustration of translator's word choice on a process of normalization

\section{Cultural - Specific Expressions versus Universal Words}

"The source-language word may express a concept which is totally unknown in the target culture. [ ...] Such concepts are often referred to as "culture-specific" Baker, M. (1992: 21). "Most "cultural' words are easy to detect, since they are associated with a particular language and cannot be literally translated [...]" Newmark, P. (1988: 95). In contrast, "Universal words [...] often cover the universal function, but not the cultural description of the referent" Newmark, P. (1988: 94). "The wheels are in 
motion.' from DVC, as an example, is not considered as CSE, but universal. It has the same meaning with 'lor moon' in Thai language and culture which means 'to set off'. In comparison, 'The wheels [...] $\therefore$ is pronounced 'lor [...]' in TL or to say they mean the same thing. According to Peter Newmark, universals are "general aspects of nature and humans and their physical and mental activities; numbers and dimensions" (1988: 103). 'The prospect of death' in 'The prospect of death is strong motivation.' which is conveyed into Thai as 'Prakan hang kwamtai' (The fortress of death) is another example of universal word from DVC. It means 'death probably or definitely happening in the future'. The TT is not a oneto-one correspondence, but a flowery language which has the same sense with the ST. Some English expressions have one-to-one correspondences in Thai, others have semantic and functional equivalents, etc.

Translation practice will be really matter when the original words are not universal but culturally specific, however, it will be easy if those CSEs in ST can be replaced with cultural equivalents in TL. For example, in translating 'A-list' in 'Collet had to admit, if there was a God, Bezu Fache would be on His A-list.' from DVC, it is replaced with cultural equivalent, 'Chan Hua Gati'. 'Chan' means 'level' whereas 'Hua Gati' is equivalent to an idiom, 'cream of the crop' in SL. The idiom means 'the best of a particular group', and 'A-list' which is a neologism means 'a list of important or prominent persons' (http://www.colinsdictionary.com). In conclusion, 'A-list' has an overlap of meaning with 'cream of the crop' as well as 'Chan Hua Kati', that is to say the CSE is replaced with a cultural equivalent in TL.

According to Peter Newmark (1988: 83), cultural equivalent is a substitution procedure of an original CSE with an equivalent one in TL which is only the approximate (e.g. Prawiro, I. Y., 2018; W, O. V. V., \& Asmarani, R., 2016). It is the procedure of translating ST into TL and TC norms, and thus it is regarded as a normalization. Azizollah Dabaghi (2012) stated in his study that most CSEs in a literary text (Blind Owl) were domesticated from Persian into English by the use of cultural equivalence, whereas Rowena Coles (2012) mentioned that normalization procedure performed under domestication strategy, and therefore cultural equivalence is a part of normalization. In case cultural equivalents of the originals do not exist in TL, other techniques will be used in normalization procedure to reduce exoticism contained in the originals to a norm of TL and TC such as the translation of 'Wicca' in 'Not only did Saunière have a personal passion for relics relating to fertility, goddess cults, Wicca, and the sacred feminine, but [...].' (from DVC) which is normalized into 'Necromancer's cult' in Thai translation. 'Wicca' refers to a religion, which spreads through England in the 1950s and subsequently through Europe and US, whose followers practice nature worship based on spiritual and occult beliefs, and call themselves 'witches', making most Western outsiders identify 'Wicca' with 'Satanism' (e.g. J. Gordon Melton, 2018). It is a cultural-specific term with a long definition. Thus, replacement with a TL word, 'Necromancer's cult' that is equivalent in function, is used to convey the richness of the original in the translation. A culture-neutral word, which is used to replace another word in SL, that does not exist in TL is functional equivalent (Newmark, P., 1988: 83). 


\subsection{METHOD AND FINDINGS}

\section{Normalization Techniques for the Translation of CSEs}

Translation technique is regarded as an instrument of textual analysis, so in the same way, normalization technique can be as an instrument for identifying and analyzing normalized text. But first of all, the concepts and definitions of existing translation techniques proposed by different scholars will be reviewed to classify normalization techniques from translation techniques. Next, different names of normalization techniques but the same concept will be gathered into one classification to get rid the confusion caused by the overlaps of terminologies and concepts between the techniques. After that, the distinguished normalization techniques will be redefined and illustrated by the examples of normalization of CSEs selected from DVC.

In 2002, by reviewing translation techniques proposed by several scholars including Vinay and Darbelnet (V\&D) (1958), Nida (1964), Vazquez Ayora (1977), Margot (1979), Newmark (1988) and Delisle (1993), Lucia Molina and Amparo Hurtado Albir reclassified 18 translation techniques that had been tested in the study of translation of cultural element in Arabic translations of A Hundred Years of Solitude by Garcia Marquez, including:

1. Adaptation: replacing a ST cultural element with one from TC. This coincides with V\&D's adaptation and Margot's cultural equivalent.

2. Amplification: adding details outside ST, e.g., explicative paraphrase and footnote. This includes V\&D's explicitation, Margot's legitimate and illegitimate paraphrase, Delisle's addition, periphrasis and paraphrase, and Newmark's explicative paraphrase.

3. Borrowing: including pure borrowing which is taking a word or expression straight from the ST without any change and naturalized borrowing which is making ST fit the TL'sspelling rules. The former corresponds to V\&D's borrowing whereas the latter, Newmark's naturalization technique.

4. Calque: literally translating a SL word or phrase and incorporating its elements into TL. This corresponds to V\&D's proposal.

5. Compensation: introducing an element or stylistic effect of ST in a new place in order that it can be reflected in TT. This corresponds to V\&D's proposal.

6. Description: replacing a term or expression with a description of its form and/or function.

7. Discursive creation: creating TT that is out of the original context to establish equivalence in translation. It corresponds to Delisle's proposal. 
8. Established equivalent: using a TL equivalent that is language in use or recognized in dictionaries. It corresponds to V\&D's equivalence and literal translation.

9. Generalization: using a more general or neutral term. It coincides with V\&D's proposal.

10. Linguistic amplification: adding linguistic elements in TT.

11. Linguistic compression: compressing linguistic elements in TT.

12. Literal translation: word for word translation which corresponds to Nida's formal equivalence, focusing on the equivalence of form, meaning, and function between a ST and its translation.

13. Modulation: lexical or structural change of ST in focus or cognitive category in TT. This coincides with V\&D's proposal.

14. Particularization: using a more concrete or precise term. This corresponds to V\&D's proposal.

15. Reduction: suppression of a ST information item in TT. This coincides with V\&D's implicitation, Delisle's implicitation and concision, and Vazquez Ayora's omission.

16. Substitution: changing linguistic into paralinguistic elements (gestures, intonation) or vice versa.

17. Transposition: changing ST's grammatical category in TT.

18. Variation: changing elements (textual tone, style, social dialect, etc.) that affect linguistic variation, such as changing tone when adapting novels for children.

Normalization technique is a technique for normalizing ST, especially CSE into TT in TL rules which will inevitably be used for readability of target readers. From all mentioned above, Adaptation and Modulation are techniques that totally incline ST towards TL rules whereas Amplification can be normalization in case of explicative paraphrase, but not in case of adding footnote. The following techniques tend towards normalization technique: Compensation (adjusting ST structure in order to be able to convey the ST in TL), Description (using TT in form of description to convey form and/or function of ST), Discursive creation (creating TT for discursive reflection of ST meaning), Generalization (using general or neutral terms in order that ST message can be conveyed), Linguistic amplification (adding TL elements in order that ST in form of TT can be understood), Substitution (changing linguistic to paralinguistic element or vice versa that is in use in TC), and Transposition (transposing linguistic items so that TT will be TL language in use). 


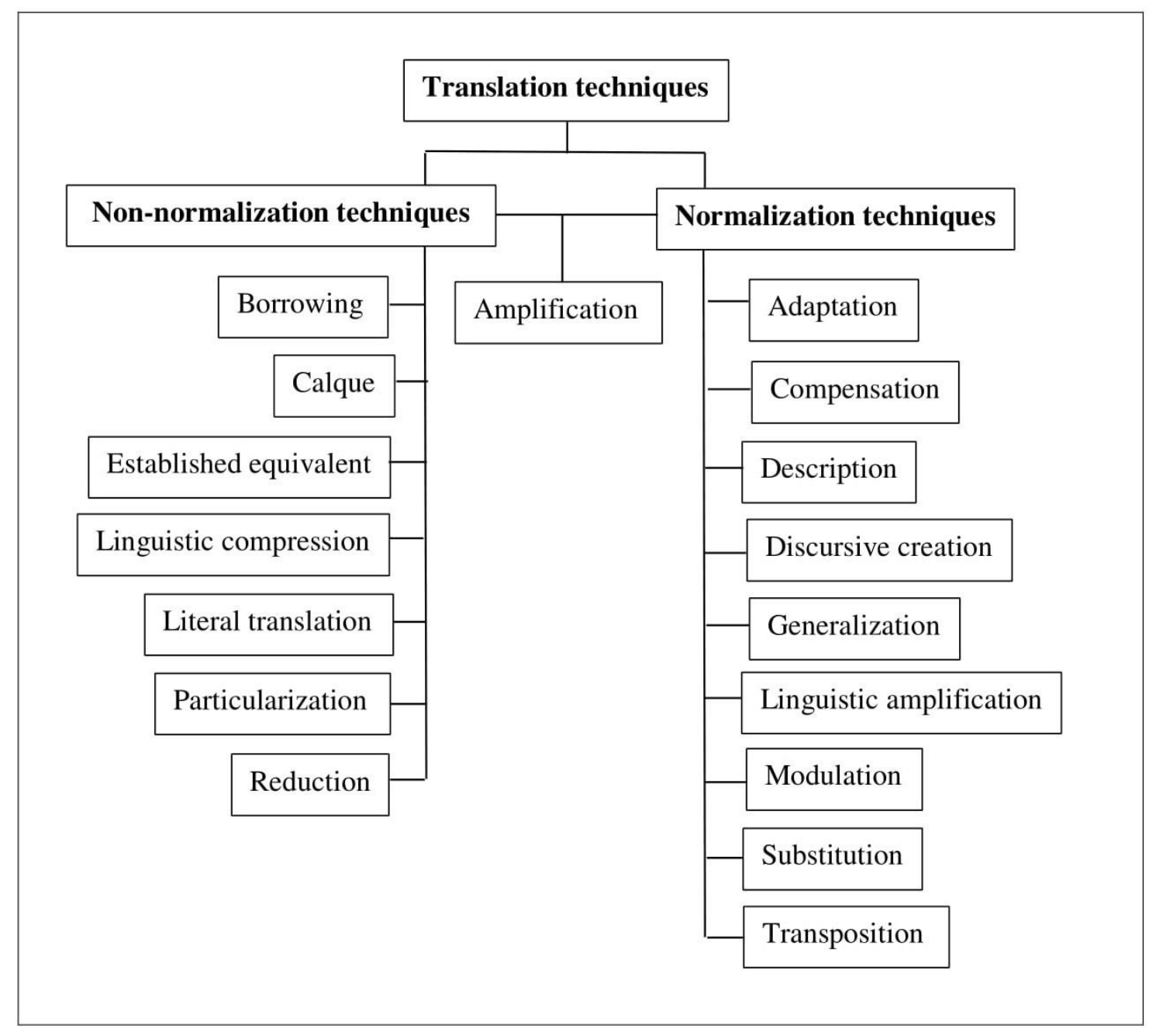

Figure 3 The distinguished normalization techniques from 18 translation techniques

Later in 2003, Malcolm Harvey used cultural-specific terms (CSTs) as a case study to present and discuss practical and ideological implications of four translation types as follows:

1. Functional equivalence: the substitution of one CST for another or the use of a referent in the TL culture whose function is similar to that of the SL referent which is appropriate for the translation of texts for the intended readers such as novels.

2. Formal equivalence: linguistic equivalence or word for word translation or a sourceoriented approach for presenting the SL system on the translation. 
3. Transcription: borrowing or transliteration, used particularly when knowledge of CSE cannot be presumed by the readers, by following with a gloss or note to elucidate significant concept, except that CSE is already clearly explained by the context.

4. Descriptive or self-explanatory translation: an appropriate strategy, used where a formal equivalent is considered insufficiently clear and where addition can be helpful to avoid ambiguity of the original SL terms.

In conclusion, Formal equivalence is SL-induced translation and subsequently Transcription is the far end of SL-oriented strategy whereas Functional equivalence has the distinguishing characteristics of normalization. For Functional equivalence, a reference in TL and TC that is well understood by TL audience and significantly similar to function of ST will be used, so it inclines to NT, as well as Descriptive or self-explanatory translation that will be used when the original cannot be literally transcribed and must be replaced by TL self-explanatory terms. After that in 2005, Jan Pedersen had investigated translation problem caused by an extralinguistic cultural-bound reference (ECR) based on Venuti'proposal of foreignizing and domesticating strategies (1995) and categorized the strategies for ECR translation as follows.

1. Official Equivalent: process of the strategy is bureaucratic rather than linguistic because the TL translation is prefabricated by official decision of people in authority. It is regarded as a standard translation, in that the TL translations are already preformed.

2. Retention: the most SL-oriented strategy, allowing ST to enter TT. The ST is marked off from the rest of the TT by quotes and occasionally by italics, but in case of a proper noun, it may be marked off in italic form. The ECR can also be adjusted slightly to TL conventions through the spelling or dropping an article. This is the far most common strategy for rendering ECRs.

3. Specification: to leave ECR untranslated in TT and add information that does not exist in the ECR to make the TT more specific through Explicitation or Addition. Explicitation in this model means adding latent material (hidden part of name) in the ECR to the TT, such as the completion of an acronym, abbreviation or official name for audience in TC who may not be familiar with the ECR. Addition means adding hidden material of sense or connotation of the ECR in TT. Through this, a guidance is given by translator's intervention to the TC audience.

4. Direct translation: to preserve original semantic load of the ECR. Connotations is not transferred or guided. In Jan Pedersen's model, this strategy is divided into Calque and Shifted. The result of claque is a stick literal translation that may appear exotic to the target readers. 
5. Generalization: replacement of an ECR referring to something specific (hyponym), with something more general (hyperonym), yet TL ECR which is more general than the ST can still retain uniqueness of the reference. It is a use of words in upper scale of hyponymy to produce TT ECR which is opposite to Addition that adds Generalization with Retention to become its result that is more specific than its original.

6. Substitution: replacing a SC ECR with a rendered transcultural ECR that could be known by the audience in TC (cultural substitute) or its paraphrase in TL. Cultural substitute is the most domesticating strategy that is fast and effective for rendering ECRs especially referring to title of official institutions. In case a TL ECR is found in bilingual dictionaries, it is an official equivalent. But if a ST ECR is not well known in TC, there will be the variability in what TL ECR will be chosen. It can be made-up substitute. Paraphrase in this model is rendering an ECR, either through a sense transfer paraphrase or a paraphrase with situational transfer in which the former is mainly used when the ST ECR is too complex for Generalization or Specification, and the paraphrase may be longer and more complex comparing to its ST whereas the latter is used when sense of SC ECR needs to be removed, and only its situational load that can be retained and paraphrased in TL.

7. Omission: replacement of a ST ECR with nothing.

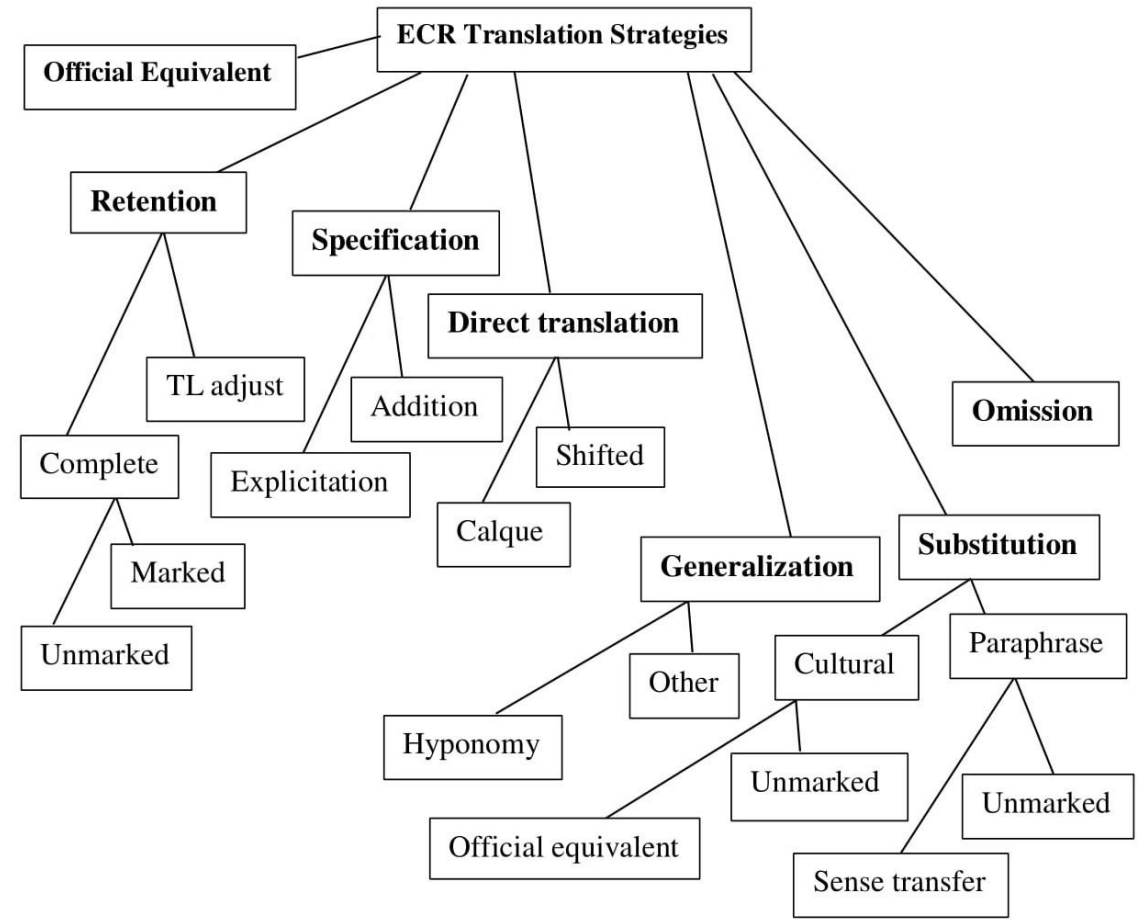

Figure 4 ECR transfer strategies by Jan Pedersen based on Venuti'proposal of foreignizing and domesticating strategies (1995) 
Referring to Jan Pedersen's statement in his book in 2011 (p.101-103), Official Equivalent is one of minimal change strategies, besides Retention and Direct Translation as more often that they retain SC ECR and add none of new material to move the ST closer to TC. In the meantime, he said that Substitution is one of the interventional strategies that supply the accessible TT for the TL audience, besides Specification and Generalization. However, Specification is not NT because it was classified as SL-oriented strategy, and even though it adds TL elements to ST, the ST itself is left untranslated in TT. So, it can be said that from seven strategies above, only Generalization, and Substitution can be categorized as NTs. Generalization is also NT as it adds hyperonym to ST ECR to produce a TT ECR that is less specific than its ST, whereas Substitution is NT since it creates target-oriented translation through the replacement of ST ECR with made-up cultural substitute or its paraphrase.

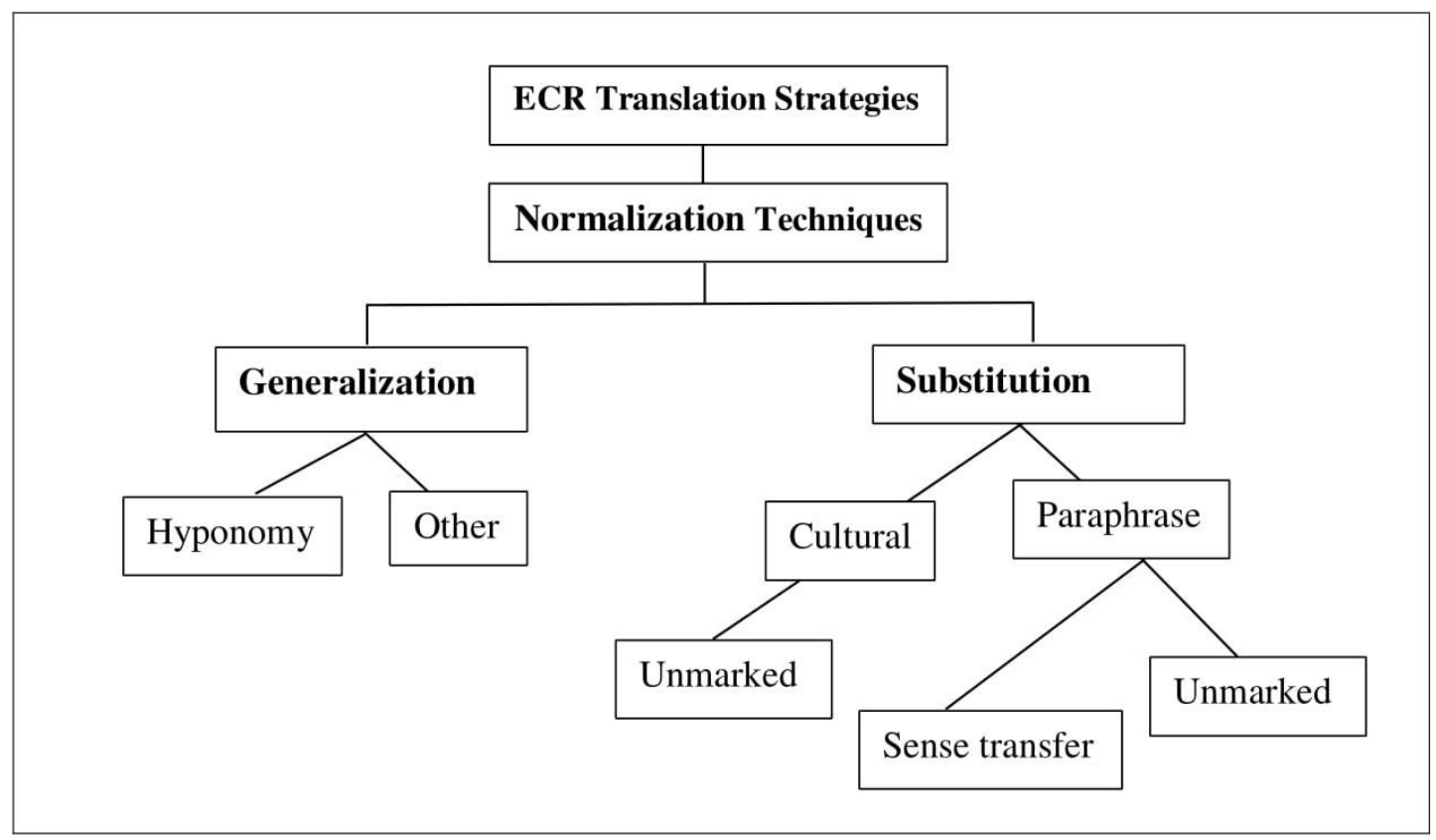

Figure 5 The distinguished normalization techniques from ECR translation strategies

The repeated NTs and concepts proposed by Lucia Molina and Amparo Hurtado Albir, Malcolm Harvey, and Jan Pedersen, are unified into one, and all NTs are then reclassified and illustrated through the examples of normalization of CSEs selected from DVC as follows.

1. Paraphrase: covering both explicative paraphrase, sub technique of amplification and modulation redefined by Lucia \& Amparo and both sense transfer paraphrase and situational transfer paraphrase by Jan Pedersen, which the former will be used when ST is too complex to 
transfer through Generalization or Specification and the latter, in case only situational load that can be transferred.

\section{Example}

'the gods of our fathers' was translated into 'god that our grandparents worship'. The ST is a figure used in Old Testament, referring to the relationship between god and his people from generation to generation, or to say, a man's god becomes god of the man's children https:/namesforgod.net/god-of-my-father/. Because the ST's literal translation is not clear enough to be understood by the TL audiences, it is transferred through an explicative paraphrase into TT without losing meaning.

2. Transposition: including compensation in which both are redefined by Lucia \& Amparo, transposing ST element to a new place in TT so that it can be TL language in use and can reflect the effect as the ST.

\section{Example}

'not part of the vocabulary' in 'Discretion was apparently not part of the vocabulary of a 15euro-per-hour watchman.' was translated into 'But a watchman who was paid 15 euro per hour wouldn't know the word 'discretion'.' The ST is generally used as 'not in someone's vocabulary' in SC which means 'not a word that someone knows and uses'. The ST functions as a verb phrase in TT to convey the ST's message in conventional TL structure.

3. Description: conveying form and/or function of ST by using TT in form of description (Lucia \& Amparo) or replacing of ST with a descriptive or self-explanatory translation (Malcolm Harvey).

\section{Example}

'The Witches' Hammer' was translated into 'Hunting the witches'. Referring to https://www.nationalgeographic.com, the ST was written in the bible in the late 15th century about hunting the witches with a real purpose to have women under control of men from Christianity. The story may be well known in SC, but not in TC. Hence, the translator chose the gist of the ST's description that can explain the meaning of itself as its TT for the TL readers' understanding.

4. Creation: creating TT for discursive reflection of ST meaning (Lucia \& Amparo). But if a ST ECR is not well known in TC, there will be the variability in what TL ECR will be chosen. It can be made-up substitute (Jan Pedersen).

Example

'Nature's divine order' was translated into 'Divine in nature' in TT. To suit the TT and target readers who believe that there are angels in nature, the TT is created by the translator based on literal ST, because the ST has complex definition, referring to belief in the order of 
nature as divine order in SC since the 5th century ACE which is not recognized by the TL readers in $\mathrm{TC}$, and because the definition is too long to put into the sentence in which the ST employed.

5. Substitution: changing linguistic to paralinguistic feature or vice versa for the familiarity with the TT among the TC audience (Lucia \& Amparo) or cultural substitute by Jan Pedersen, replacing an ECR with a made-up cultural substitute which is similar to adaptation by Lucia \& Amparo that inclines ST towards TC by replacing it with cultural element chosen from TL.

\section{Example}

'a subtle thumbing of his nose' an idiom of paralinguistic feature, a gesture of insulting was translated into 'derision' in TL. Because it is not commonly used among the TL audience in TC, there is none of TL idiom reflecting the same result. Thus, the paralinguistic feature is replaced by linguistic feature in form of a generic word.

6. Generalization: using general or neutral terms to convey ST message (Lucia \& Amparo) or replacement of an ECR using specific terms with more general TL terms to retain uniqueness of the reference (Jan Pedersen)

\section{Example}

'the birds-and-the-bees' was translated into 'sexology'. The ST is an idiomatic expression, generally used in SC for referring to sexual intercourse for explaining to the kids. Its meaning and use are not universally but culturally specific. So, for the TL readers' understanding, the ST's message is conveyed through the general TL term.

7. Linguistic amplification: adding TL elements in order to transfer ST into understandable TT (Lucia \& Amparo)

\section{Example}

'a not so subtle howdy-do' in 'A not so subtle howdy-do to the boys in Rome. They might as well have resurrected Stonehenge in downtown London.' was translated into 'it is probably not about to impress'. Referring to www.freedictionary.com, the phrase is primarily heard in US as 'a fine howdy-do', meaning 'an unpleasant affair'. The original in a phrase form was transferred into a TL sentence in order that it can be conveyed and reflect sense and meaning of the original.

8. Functional equivalence: a distinguishing NT, the use of a TL referent that is significantly similar to function of ST and well understood in TC by TL audiences (Malcolm Harvey). 


\begin{abstract}
Example
'schlockmeister' was translated into 'slapdash person' in TT. Originally used in the 20th century, the ST, an informal North American, means a person who deals in cheap or trashy goods (www.lexico.com), as a slang, it refers to a filmmaker, writer, etc. who produces kitsch (www.collinsdictionary.com). There is none of TL cultural substitute or official equivalent of the ST, there is only functional equivalent that can function in TT to reflect similar results to the ST.
\end{abstract}

As illustrated above, there may be relation between each CSE and each unified NT. The CSE which is normalized through Paraphrase, is composed of a group of words (phrase) that is normally used and well understood by users in SC, but needs more explanation for readers in TC. However, to make it domestic and understandable, not by adding some TL elements and using specific TL terms, but reiterate it all in TL version based on sense of the ST. The CSE, normalized through Transposition, cannot be understood by TL readers because they say another different way for the same meaning. Somehow, the translator did not use TL cultural equivalent for it. Thus, its position is moved to the place that fits to TL structure, so that its meaning can be conveyed. The CSE, normalized through Description, is a noun phrase referring to event dating back to hundreds of years in SC. So, of course, translated text of the ST's long historical description needs to be concise to conserve form but still contain function of the original. Likewise, the CSE, normalized through Creation, is a noun phrase with complex explanatory definition, but more than that, it is about belief which is similar but yet different from belief in TC, so the technique's result is a made-up translation for accessibility of TL readers, however, similar to its original. For Substitution technique, its result is a general term, replacing an idiom of paralinguistic feature, as the ST is neither used nor recognized in TC, it is unavoidably to convey meaning of the gesture with linguistic feature. This is different from case of Generalization that the neutral word is used as TT in order to cover the CSE's meaning as a reason for propriety, while the CSE in a phrase form is necessary to be returned into a sentence by Linguistic Amplification, in order that it can fit with the other content words in the sentence, and can reflect the ST's sense and meaning. Functional Equivalence, the last unified technique, normalizes a noun, functioning as a slang which has quite a wide meaning, and has no TL official equivalent yet in dictionaries, so the TT, carrying the CSE's functional meaning is chosen to fit with context.

As can be seen, each NT fits each CSE's needs. The selected CSEs from DVC to illustrate the normalization process of the eight unified NTs, are in form of word or phrase, but their TTs are in form of word, phrase or even sentence. Form of the original does not restrict form of its TT as well as its NT. From the examples, the CSE normalization process occurs in lexical level through the use of every NT except transposition and linguistic application in which the process occurs in syntactic level. It probably can be said that the NTs restrict their level of process and form of TT. Through translation analysis, the cultural reference of CSE restricts everything: NT and TT form. In order that it can be reflected in TL, and its TT can conserve and convey the original properties, the suitable NT will be used to transform it into TT in TL norms such as syntactic structure and proper wording. From analysis of previous studies, CSEs that need to be normalized in the translation process are the idiomatic or figurative expressions (e.g., proverb, idiom, pun, and sarcasm) that word order and constituent structure are fixed, by which their meanings or senses are not literally reflected, allusions 
or proper nouns/phrases, culture-specific nouns/compound nouns, or neologisms with complicated definitions, culture-specific references, and moreover, histories that are well known only among speakers in SC. In contrast, normalized CSEs are not footnotes, amplifications in parentheses, borrowed words, formal equivalents or established equivalent, recognized in bilingual dictionaries. Through normalization process, the followings are regarded as normalized CSEs.

1. Paraphrase, made by explicatively reiterating ST in TL norm

2. Translation, made by rearranging ST elements in TL structure into TL language in use

3. Descriptive or self-explanatory translation

4. Made-up translation which discursively reflects its ST's meaning

5. Cultural substitute, chosen by translator's subjectivity from variability of TL ECR

6. Translation, made by the use of more general or neutral terms than its original

7. Linguistic element that is used to substitute an unknown paralinguistic element to the target readers

8. Functional equivalent

\subsection{CONCLUSION AND FUTURE WORK}

Although domestication and normalization have common purpose of shifting foreign ST closer to TL and TC of target readers, domestication runs through normalization process, so it can be said that the latter is under the former and it can be regarded as a technique of the former one. Normalization has been intervened by translator's subjectivity in choosing words to create ST in TL version. It cannot be denied that translator's word choice is a part of normalized text. And NT used in normalizing CSE, is restricted by characteristics of CSE itself to make it as normal TL in use as possible. This research based on literature review reveals what normalization is, how it is different from domestication, and how it works in which all answers lead to revealing of normalization techniques which are particularly use for translating CSE which is the main problem of all kinds of translation, especially literature. How it goes on normalization process which is explained through the illustration of normalization of CSE selected from DVC, significantly shows the relevance between NT and characteristics of CSE which will be as a guidance of how to solve translation problem of CSE. This will break down difficulties of CSE translation and will be beneficial for translation industry.

It does not mean that normalization is only used for CSE translation, but CSEs need normalization to transfer their properties into TT. And the use of any techniques to transcribe ST in order that its message can be conveyed in TL and sounds natural, its outcome or TT tends to be normalized text. A CSE is any expression that its reference is known and used among the SL users to 
express any concept that they are immediately aware, in which to express the same concept, people in other TCs have other different references and say in other different ways. That is why a CSE can be substituted by any cultural equivalents in other TLs, and cultural equivalence is as a NT, because it makes the CSE normal like TL text. Based on DVC, cultural equivalence is the most-used NT. This can be understood that although come from different cultures and use different languages, as human beings on earth, they have a lot of the same concepts. The normalization of a CSE is related to characteristics of the CSE itself which will restrict any NT to choose or create a substitute for it, and unavoidably it is related to translator's subjectivity. The normalization process's result reflects as equal effect to its ST as possible as a normalized text or a made-up translation which is a matter of course that normalization study will not involve official equivalence. In the sooner future, the normalization study may lead to the new more formal or established definition of CSEs in bilingual dictionaries, and the difficulties of CSE translation will then be more and more lessened. However, the research about factors that influence the use of the NTs and its outcome should be studied in a wider range for wider knowledge about the normalization.

\section{REFERENCES}

Al-Idhesat, T. A. S. 2016. The Translation of Cultural Elements from English into Arabic in Oliver Twist. Master Degree. University of Malaya, Kuala Lumpur. Retrieved from http://studentsrepo.um.edu.my/id/eprint/6813.

Baker, M. 1992. In Other Words: A Coursebook on Translation. 1st ed. London and New York: Routledge.

Baker, M. 1993. Corpus Linguistics and Translation Studies - Implications and Applications. In M. Baker, G. Francis, \& E. Tognini-Bonelli (Eds.). Text and Technology: In Honour of John Sinclair . Philadelphia and Amsterdam: John Benjamins Publishing Company. 233-250.

Baker, M. 1996. Corpus-based Translation Studies: The Challenges that Lie Ahead. In H. Somers (Ed.). Terminology, LSP and Translation: Studies in Language Engineering, in Honour of Juan C. Sager. Amsterdam and Philadelphia: John Benjamins Publishing Company. 18: 175186.

Coles, R. 2012. Domesticating the Trauma of War in Translation. Libri \& Liberi: ̌̌ asopis za istraživanje dječ je književnosti i kulture. 1(1): 45-66.

Dabaghi, A., \& Bagheri, M. 2012. The Issue of Translating Culture: A Literary Case in Focus. Theory and Practice in Language Studies. 2(1): 183-186. Doi:10.4304/tpls.2.1.183-186.

Giaber, J. M. 2015. Implications of Lexical Choices in Translating Novels as Literary Texts: The Case of A Farewell to Arms in Arabic trans-kom. 8(2): 415-445.

Halliday, M. A. K. 1985. An Introduction to Functional Grammar. London: Edward Arnold.

Harvey, M. 2003. A Beginner's Course in Legal Translation: The Case of Culture-Bound Terms.

Marco, J. 2009. Normalization and the Translation of Phraseology in the COVALT Corpus. Meta. 54(4), 842-856. Doi: https://doi.org/10.7202/038907ar. 
Melton, J. G. 2018, October 08, 2018. Wicca. Retrieved from https://www.britannica.com/topic/Wicca.

Molina, L., \& Albir, A. H. 2002. Translation Techniques Revisited: A Dynamic and Functionalist Approach. Meta. 47(4): 498-512. Doi: https://doi.org/10.7202/008033ar.

Mostafa, M. M. 2015. Translating Le Monde and El País English Business Loan Terms into Arabic: A Corpus-based Analysis. Journal of Language Teaching and Research. 6(1): 61-70. Doi: http://dx.doi.org/10.17507/jltr.0601.07.

Mussche, E., \& Willems, K. 2010. Fred or farī d, bacon or bayḍ un ('egg')? Proper Names and Cultural-specific Items in the Arabic Translation of Harry Potter. Meta. 55(3): 474-498. Doi: https://doi.org/10.7202/045066arCopiedAn.

Newmark, P. 1988. A Textbook of Translation. New York and London: Prentice-Hall International

Nida, E. A. 1964. Toward a Science of Translating: With Special Reference to Principles and Procedures Involved in Bible Translating. Leiden: E. J. Brill.

Olexová, K. 2009. Translation of Character Names in Children's Literature: A Chronological View. Master Diploma Thesis. Masaryk University.

Pedersen, J. 2005. How is Culture Rendered in Subtitles? Paper Presented at the MuTra 2005 Challenges of Multidimensional Translation.

Pedersen, J. 2011. Subtitling Norms for Television: An Exploration Focussing on Extralinguistic Cultural References (Vol. 98). Amsterdam and Philadelphia: John Benjamins Publishing.

Prawiro, I. Y. 2018. Translation: When the Language, Culture and Herbs Are Mixed in the Same Bowl. Wacana Didaktika. 10(2): 22-30.

Shuttleworth, M., \& Cowie, M. 1997. Dictionary of translation studies. London and New York: St. Jerome Pub.

Venuti, L. 1995. The Translator's Invisibility: A History of Translation (S. Bassnett \& A. Lefevere Eds.). London and New York: Routledge.

Vinay, J.-P., \& Darbelnet, J. 1995. Comparative Stylistics of French and English: A Methodology for Translation. Amsterdam/Philadelphia: John Benjamins Publishing Company.

Vinay, J.-P., \& Darbelnet, J. 1995. Introduction. In J. C. Sager \& M.-J. Hamel (Eds.). Comparative Stylistics of French and English: A Methodology for Translation. Amsterdam/Philadelphia: John Benjamins Publishing Company. 11: 30-41.

W, O. V. V., \& Asmarani, R. 2016. The Translation Procedures of Cultural Expressions Applied in "A Game of Thrones". LITE. 12(2): 199-218.

Wang, F. 2014. An Approach to Domestication and Foreignization from the Angle of Cultural Factors Translation. Theory and Practice in Language Studies. 4(11), 2423-2427. Doi:10.4304/tpls.4.11.2423-2427,

Xia, Y. 2014. Normalization in Translation: Corpus-based Diachronic Research into Twentieth-century English-Chinese Fictional Translation. Newcastle upon Tyne, UK: Cambridge Scholars Publishing.

Yang, W. 2010. Brief Study on Domestication and Foreignization in Translation. Journal of Language Teaching and Research. 1(1): 77-80. Doi:10.4304/jltr.1.1.77-80. 